\title{
An explanation of the $25 \%$ male excess mortality for all children under 5
}

\author{
David T. Mage ${ }^{1,2}$, E. Maria Donner ${ }^{3}$
}

'World Health Organization (retired)

${ }^{2}$ U.S. Environmental Protection Agency (retired)

${ }^{3}$ Dupont Haskell Global Centers for Health and Environmental Sciences, Newark, DE, USA, magedonner@aol.com

\begin{abstract}
:
BACKGROUND: To demonstrate that an epidemiologic probability model of a hypothesized X-linkage for Sudden Infant Death Syndrome (SIDS) that predicted its $50 \%$ male excess, also predicts the $25 \%$ male excess of all child mortality for ages under 5 years. METHODS: Neglecting trauma, infants die naturally from either respiratory causes $\mathrm{R}$ (breathing stops first) or cardiac causes $\mathrm{C}$ (heart stops beating first). An hypothesized dominant $X$-linked allele with frequency $p=1 / 3$, that is protective against acute anoxic encephalopathy, predicted the $50 \%$ male excess of R. Given the $\sim 0 \%$ male excess for cardiac deaths $C$, and assuming equal death risk for females by $R$ and $C$, their average predicts a $25 \%$ male excess for equal numbers of infant males and females at risk. Thus, 5 males would die for each 4 females dying from all causes, predicting a male fraction of $5 / 9=0.55556$.

RESULTS: Vital statistics for gender of children under 5 years at risk of dying and their corresponding mortality are obtained from the U.S.A. and multiple European countries. For 17 data sets from 15 countries, we total over 1.2 Billion child-years at risk and over 2.6 million child deaths. The observed total under 5 year male fraction, correcting for the nominal $5 \%$ male livebirth excess, is 0.55633 , virtually as predicted.

CONCLUSIONS: An X-linked dominant allele protective against respiratory failure, predicts accurately the $5 / 9$ male fraction of all child mortality under 5 years. DNA study of SIDS can identify the candidate X-linked gene locus.

Keywords:
\end{abstract}

SIDS, Respiratory Arrest, Cardiac Arrest, X-linkage

\section{BACKGROUND}

Although the higher rate of male mortality in infancy was well known, Naeye et al. [1], were the first to propose that this male excess was caused by an $\mathrm{X}$-linkage. They analyzed neonatal mortality in the first 72 hours of life and concluded: "The biologic difference must originate in the genetic differences between the sexes and those genetic differences are a consequence of disparity in the number of the $\mathrm{X}$ chromosomes...a given male may possess only one while a female may possess two, one of which will be active in some of her cells and the other active in occasional other cells...This gives the female options for variability not open to the male"[1]. In 1996 we found that Sudden Infant Death Syndrome (SIDS) and other causes of respiratory deaths in infancy had a relatively constant $50 \%$ male excess for equal numbers of males and females at risk in all mortality datasets from throughout the developed world $[2,3]$. We realized that an X-linkage to a gene with a $p=1 / 3$ dominant allele protective against respiratory failure and a corresponding non-protective $q=2 / 3$ recessive allele would predict the $50 \%$ male excess of SIDS and all other respiratory deaths. The XY male would be at risk with frequency $q=2 / 3$ and the XX female would be at risk with frequency $q^{2}=4 / 9$ giving the observed ratio $q / q^{2}=6$ males to 4 females. For a $5 \%$ male birth excess this predicted the observed SIDS male fraction of $0.612=\left(6^{*} 1.05\right) /$ $\left(6^{*} 1.05+4\right)$. [2] In 2004 we revisited our model and retested it with new mortality data that became available on line and in the literature [4,5]. We showed, for equal numbers of males and females at risk, that virtually all infant respiratory deaths still had a 50\% male excess and all infant cardiac deaths still had a $0 \%$ male excess, and that infant mortality under one year from all causes still had a $25 \%$ male excess [4-6]. Now in this paper we show this $25 \%$ male mortality excess in infancy extends beyond one year up to 5 years and is similar for all developed countries, with different racial mixes and medical systems. This commonality is not in the pediatric literature and represents a surprising new finding.

\section{DATA ANALYSIS}

Let $\mathrm{M} \mathrm{XY}$ male and $\mathrm{F} X X$ female children be at risk of dying between birth and their 5th birthday, with $M=F$. As Guntheroth [7] pointed out, all infant deaths have only one degree of freedom, and are either from $R=$ respiratory arrest (breathing stops first) or $\mathrm{C}=$ cardiac arrest (heart stops first), except for unusual cases, such as a fall from a great height, where both cease simultaneously. He noted that virtually all SIDS and other respiratory deaths display unclotted blood in the heart at autopsy, inferring "that circulation persisted in an hypoxic state [7]." The $50 \%$ male excess of infant $R$ is explainable by an $X$-linked dominant allele protective against $R$ with frequency $p=1 / 3$ as shown above. The absence of an X-linkage protection against cardiac deaths in infancy would explain their observed $0 \%$ male excess for $C$. If all female infants have an equal probability of dying from either $\mathrm{R}$ or $\mathrm{C}$ then the average male excess of all infant deaths would be $25 \%$, the average of the $50 \%$ male excess in $\mathrm{R}$ and the $0 \%$ male excess in $\mathrm{C}$. This is equivalent to assuming that the probability $p(R)$ in all infant mortality is equal to the probability that all the infants at risk are susceptible to $R$, so $p(R)$ $=\left(q M+q^{2} F\right) /(M+F)=5 / 9$ and, by definition, $p(C)=1-p(R)=4 / 9$. Thus a total of 5 male child deaths are predicted for every 4 female child deaths resulting in a predicted total male fraction of $5 / 9=0.55555 \ldots$ written as 0.55556 for equal numbers of males and females at risk. 
In practice there are not an equal number of male and female children at risk $(M \neq F)$ because there is a nominal $5 \%$ male livebirth excess so that $M / F$ $\approx 1.05$ at birth (see Table 1). However we note that if $m$ male and $f$ female children under 5 years die, the death rates can be normalized by dividing by the numbers of males $\mathrm{M}$ and females $\mathrm{F}$ at risk. Thus the expression $(\mathrm{m} / \mathrm{M}) /$ $(m / M+f / F)=5 / 9$ represents the predicted relationship correcting for the male excess birth rate that we test in the following section.

\section{RESULTS:}

Table 1 shows the vital statistics for infants and small children under 5 years of age by 17 data sets from 15 different countries listed in order of increasing numbers of deaths [8-14]. There are data for a total over 1.2 billion infant-years at risk and over 2.6 million male and female deaths. The overall normalized male fraction is 0.55633 , almost exactly the same as 0.55630 observed for the U.S. (Table 2), corresponding to the value predicted by our X-linkage model of 0.55556 [14]. We note that the observed total male fractions for these 12 European countries are all very similar, and in fact some are virtually identical (e.g., U.K. $=0.55292$; France $=0.55324$; Finland $=0.55334$; Norway $=0.55343$ ) which cannot occur by chance alone [9]. The Eurostat website http://epp.eurostat.ec.europa.eu/ portal/page/portal/statistics/themes [9] provided us these European data on annual numbers of male and female infants less than 5 years of age at risk ( $\mathrm{M}$ and $\mathrm{F}$ ) under the theme of Population, and annual data on numbers of males and females less than 5 years of age dying from all causes ( $m$ and f), under the theme of Health.

Table 1. Summary of Total Infant and Child Mortality under 5 Years Old [8-14]. Predicted Male Mortality Fraction $=(\mathrm{m} / \mathrm{M}) /(\mathrm{m} / \mathrm{M}+\mathrm{f} / \mathrm{F})=0.55556$

\begin{tabular}{|c|c|c|c|c|c|c|}
\hline Country & Years & Males at Risk (M) & Females at Risk (F) & Male Deaths $(m)$ & Female Deaths ( $f$ ) & Male Fraction \\
\hline Japan [8] ${ }^{a}$ & 2013 & - & - & $m / \mathrm{M}=0.0024$ & $f / F=0.00192$ & 0.55556 \\
\hline Norway[9] & $1994-2010$ & $2,311,252$ & $2,174,336$ & 2,462 & 1,869 & 0.55343 \\
\hline Norway [10] & 1967-1998 & 637,055 & 604,163 & 3,103 & 2,344 & 0.55663 \\
\hline Switzerland [9] & $1994-2010$ & $3,411,320$ & $3,228,990$ & 2,839 & 2,151 & 0.55542 \\
\hline Denmark [9] & $1994-2010$ & $2,908,314$ & $2,766,921$ & 3,225 & 2,512 & 0.54983 \\
\hline Austria [9] & $1994-2010$ & $3,678,152$ & $3,499,533$ & 4,261 & 3,323 & 0.54955 \\
\hline Finland [9] & $1985-2012$ & $4,375,607$ & $4,191,260$ & 4,277 & 3,307 & 0.55334 \\
\hline Australia [11] & $2002-2012$ & 442,607 & 419,580 & 7,521 & 5,780 & 0.55228 \\
\hline Netherlands [9] & $1994-2010$ & $8,535,367$ & $8,145,219$ & 10,878 & 8,351 & 0.55418 \\
\hline Sweden [12] & $1968-2013$ & $12,468,164$ & $11,829,786$ & 18,814 & 14,072 & 0.55919 \\
\hline Ireland [9] & $1960-2012$ & $8,384,081$ & $7,961,972$ & 26,970 & 20,586 & 0.55440 \\
\hline France [9] & $1998-2012$ & $29,878,412$ & $28,508,178$ & 29,925 & 23,057 & 0.55324 \\
\hline Eng \& Wales[13] $]^{\mathrm{ab}}$ & 1969-1976 & $2,870,265$ & $2,734,688$ & 22,965 & 17,502 & 0.55556 \\
\hline U.K.[9] & $1993-2012$ & $37,512,290$ & $35,746,638$ & 49,253 & 37,949 & 0.55293 \\
\hline Belgium [9] & $1960-2012$ & $17,439,354$ & $16,615,713$ & 58,183 & 42,504 & 0.56602 \\
\hline Germany [9] ${ }^{\mathrm{c}}$ & $1985-2012$ & $53,117,609$ & $50,411,223$ & 73,116 & 55,199 & 0.55695 \\
\hline USA [14] & $1968-2013$ & $434,495,355$ & $415,309,285$ & $1,206,923$ & 920,109 & 0.55630 \\
\hline Totals [9-14] & Per Above & $622,465,204$ & $594,147,485$ & $1,524,715$ & $1,160,615$ & 0.55633 \\
\hline
\end{tabular}

aData are for infants under 1 year of age.

${ }^{b}$ Data given as $m, f$ and $m / M, f / F$

cUntil 1990 former FRG.

Table 2. Summary of U.S. Total Infant and Child Mortality under 5 Years Old, 1968-2013. http://wonder.cdc.gov/mortSQL.html [14]. Predicted Male Mortality Fraction $=(\mathrm{m} / \mathrm{M}) /(\mathrm{m} / \mathrm{M}+\mathrm{f} / \mathrm{F})=0.55556$

\begin{tabular}{|c|c|c|c|c|c|}
\hline Years & Males at Risk (M) & Females at Risk (F) & Male Deaths $(m)$ & Female Deaths $(f)$ & Male Fraction \\
\hline $1968-1978$ & $93,909,018$ & $89,934,399$ & 439,853 & 328,657 & 0.56173 \\
\hline $1979-1998$ & $188,728,193$ & $180,087,206$ & 501,132 & 383,578 & 0.55489 \\
\hline $1999-2013$ & $151,858,144$ & $145,287,680$ & 265,938 & 207,874 & 0.55035 \\
\hline $1968-2013$ & $434,495,355$ & $415,309,285$ & $1,206,923$ & 920,109 & 0.55630 \\
\hline
\end{tabular}




\section{DISCUSSION}

The consistent overall male fraction of child mortality under 5 years of age as shown in Table 1 is predicted based on only a single parameter of $p=$ $1 / 3$ for the frequency of an X-linked dominant allele at a presently unknown gene locus that is protective against respiratory failure. We reasoned that this common $50 \%$ male excess and age distribution for sudden $\mathrm{R}$ implied that there must be a common terminal mechanism for all the different causes of respiratory death [15]. We proposed that this commonality arises from a dominant $p=1 / 3$ allele that codes for a presently unknown enzyme allowing respiratory control neurons in the brainstem to shift from aerobic oxidation to anaerobic oxidation to maintain their life when the blood oxygen tension they measure falls below a critical level. Those children with only the recessive allele with frequency $q=2 / 3$ would succumb to an acute anoxic encephalopathy, such as in SIDS, if, and only if, they have a combination of gender-independent risk factors that combine to reduce the blood's exiting cerebral oxygen tension to below that critical level. For example, an undetected prodromal low-grade respiratory infection in such a genetically susceptible infant with undetected severe physiological anemia (not measured at autopsy because of lividity) and neurological prematurity can explain the age and gender distributions of SIDS [16-19].

In our epidemiologic analyses we consider the vital statistics data in Tables 1 and 2 as a probability sample from an infinite population, subject to the following assumptions:

- All infants are either $X X$ or $X Y$ : we neglect multiple- $X$ syndromes such as $\mathrm{XXX}, \mathrm{XXY}, \mathrm{XX}$ male, etc.

- All child deaths are natural: we neglect possibility of gender-dependent infanticide, etc.

- All child deaths are independent from each other: we neglect communicable disease transfer from one infant to another who both die from it.

- All deaths are first either respiratory arrest or cardiac arrest: we neglect simultaneous cessation (e.g., trauma as in a fall from great height) [7].

- All deaths in years cited were born in same years: For example, defining an infant < 1 year death rate in 2012 by dividing the number of deaths in 2012 by the number of births in 2012 is only an approximation. This is because some of the infants dying in 2012 were born in 2011 and some of the infants born in 2012 died in 2013. We neglect the differences in these quantities and assume they balance out.

- Male hyperactivity is not yet an excess mortality risk factor $<5$ years. We assume prior to 5 years of age that the infants and children are always under direct supervision of a parent or other adult while at home or in day-care. After 5 years male children begin to express hyperactivity relative to that of the female of identical age, and may play independently of direct adult supervision involving more risky activity than the comparative females.

- Only the sampling error of a finite sample size is involved. We assume that there is no measurement or transcription error in recording the infant gender.

Any violation of these assumptions is expected to increase the variance of these data and contribute to the difference between the predicted and observed values if the model is correct. We suggest that the difference of $0.2 \%$ we observed between 0.55556 and 0.55633 may be because of sampling error and the additional variance due to the known violations of these assumptions.

\section{CONCLUSION}

The consistent closeness of fit between the observed male fraction of 0.55633 and the predicted 0.55556 value is strong support for the claim by Naeye et al. [1] that male excess infant mortality must [emphasis added] be $\mathrm{X}$-linked. There is no other explanation, to our knowledge, for the ability of a hypothesized single non-protective $X$-linked recessive allele with frequency $q=2 / 3$ to predict the 0.61 male fraction of SIDS as well as the $5 / 9$ male fraction of all child mortality under 5 years from all causes.

We propose that an analysis of the DNA of SIDS cases and controls can identify the X-linked dominant allele that is missing in all SIDS (assuming no false positive cases) and present in $1 / 3$ of the live male controls. If the hypothesized enzyme this dominant allele codes for can be identified, and it passes through the blood-brain barrier, then prophylaxis by its administration may be possible to prevent $5 / 9$ of all infant and child mortality.

\section{REFERENCES}

[1] Naeye RL, Burt LS, Wright DL, Blanc WA, Tatter D. Neonatal mortality, the male disadvantage. Pediatrics 1971; 48: 902-906.

[2] Mage DT, Donner EM. A genetic basis for the sudden infant death syndrome sex ratio. Med Hypotheses 1997; 48: 137-142.

[3] Mage DT, Donner EM. An X-linked genetic susceptibility for SIDS and respiratory failures. J Sudden Infant Death Syndrome Infant Mortality 1996 ;1: 295-305.

[4] Mage DT, Donner EM. The fifty percent male excess of infant respiratory mortality. Acta Paediatrica 2004; 93: 1210-1215.

[5] Mage DT, Donner EM. The X-linkage hypothesis for SIDS and the male excess in infant mortality. Med Hypotheses 2004; 62: 564-567.

[6] Mage DT, Donner EM. Is excess male mortality from sudden infant death syndrome and other respiratory diseases X-linked? Acta Paediatrica 2014; 103 : 188-193.

[7] Guntheroth WG. Crib Death: The Sudden Infant Death Syndrome. 3rd ed. Futura Publishing Company. Armonk. 1995. pp 45, 229.
[8] CIA World Fact Book, 2013. http://www.indexmundi.com/japan/infant_mortality_ rate.html

[9] European Commission, Eurostat, 2014. http://epp.eurostat.ec.europa.eu/portal/ page/portal/statistics/themes Accessed July 31, 2014

[10] Øyen N, Irgens LM, Skjaerven R, Morild I, Markestad T, Rognum TO. Secular trends of SIDS in Norway 1967-1988: application of a method of case identification to Norwegian registry data. Paed Perinatal Epidem 1994;8:263-281.

[11] Australia Bureau of Statistics, 2014. http://www.abs.gov.au/AUSSTATS/

[12] Statistics Sweden, 2014. http://www.statistikdatabasen.scb.se

[13] Carpenter RG, Gardner A. Variations in unexpected infant death rates relating to age, sex and season. Studies on Medical and Population Subjects No. 45, HMSO, London, 1982.

[14] Centers for Disease Control and Prevention, National Center for Health Statistics. Compressed Mortality Files 1968-1978, 1979-1998 and 1999-2013 on CDC WONDER Online Database. Accessed at http://wonder.cdc.gov/mortSQL.html on March 5, 2015. 
[15] Mage DT, Donner EM, Vennemann M, Fleming P, Sol-Church K, Drake R, Gulino SP. All sudden infant respiratory deaths may result from the same underlying mechanism. Scand J Forensic Sci. 2012; 18(1):2-10.

[16] Poets CF, Samuels MP, Wardrop CAJ, Picton-Jones E, Southall DP. Reduced haemoglobin levels in infants presenting with apparent life threatening events - a retrospective investigation. Acta Paediatrica 1992; 81:319-321.
[17] Gupta R, Helms PJ, Jolliffe IT, Douglas AS. Seasonal variation in sudden infant death syndrome and bronchiolitis - A common mechanism? Am J Resp Critical Care Med. 1996; 154: 431-435.

[18] Mage DT. A probability model for the age distribution of SIDS. J Sudden Infant Death Syndrome Infant Mortality 1996; 1: 13-31.

[19] Mage DT, Donner M. A unifying theory for SIDS. Intl J Pediat 2009; 2009: 368270. 